\title{
Immunologic Pattern of Hepatitis B Infection among Exposed and Non- Exposed Babies in A PMTCT Program in Low Resource Setting: Does Every Exposed Newborn Require 200IU of Hepatitis B Immunoglobulin?
}

\section{Onakewhor $\mathrm{JUE}^{1,2 *}$, Charurat $\mathrm{M}^{2}$, Matthew $\mathrm{O}^{3}$, Esosa Osagie ${ }^{4}$, Asemota $\mathrm{MO}^{4}$ and Omoigberale $\mathrm{A}^{5}$}

+ Department of Obstetrics and Gynecology, University of Benin Teaching Hospital, Benin City, Nigeria

${ }^{2}$ Institute of Human Virology, University of Maryland School of Medicine, Baltimore, Maryland, United States of America

${ }^{3}$ Research Department, Institute of Human Virology, Nigeria, Abuja Nigeria

${ }^{4}$ Department of Laboratory, University of Benin Teaching Hospital, Benin City, Nigeria

${ }^{5}$ Department of Child Health, University of Benin Teaching Hospital, Benin City, Nigeria

\begin{abstract}
Background: Hepatitis B (HBV) is a vaccine-preventable infection. Vaccination programs instituted for virtual elimination of vertical and horizontal transmission are rarely evaluated. The prohibitive cost of HBV immunoprophylaxis (HBIG) engenders restrictive access in low-resource settings.
\end{abstract}

Objective: Comparison of the immunologic pattern of HBV seromarkers and mother-to-child transmission (MTCT) rates among exposed and non-exposed babies who received either standard 200 iu or 100 iu HBIG in resource low settings.

Method: This prospective pilot observational study involved a cohort of HBV-infected pregnant women and exposed babies and HBV-uninfected women-baby pairs as control at the Department of Obstetrics and Gynecology, UBTH, Nigeria. HBV seromarkers were detected using rapid, direct, third generation immunochromatographic test for qualitative monoclonal and polyclonal anti-HBsAg antibodies. Reactive samples were reanalyzed using LumiQuick HBV-5 panel test for visual detection of HBs-antigen, anti-HBs-antibodies, HBe-antigen, anti-HBe antibodies, anti$\mathrm{HBc}(\operatorname{lgG} / \operatorname{lgM})$ antibodies. Reactive samples were confirmed with ELISA. Exposed babies were "self-selected" into receiving either $200 \mathrm{IU}$ or $100 \mathrm{IU} \mathrm{HBIG}$ within 12 hours of birth plus 3-dose course of HBV vaccine. Outcome measures were incidence of MTCT of HBV, pattern of immunologic response for HBV seromarkers and proportion of vaccine non-responders.

Results and conclusion: The MTCT rate was $0.0 \%$ for all groups with no homogenous pattern of maternal to fetal transfer of HBV seromarkers. The overall rate of non-response to vaccination was high $(8.0 \%)$ with exposed infants being poorer responders $(17.1 \%)$ to vaccination $p<0.01$. We advocate introduction of HBIG as integral part of care into the NPI program and multicenter studies to evaluate our findings before policy change.

Keywords: Hepatitis B; PMTCT; Seromarkers; Immunology; MTCT; HBIG

\section{Introduction}

Hepatitis B (HBV) is a ubiquitous DNA virus. Globally, an estimated 2 billion people or one in three have a marker for past or current infection [1], About 4.5 million new infections occur each year and one in four progress to liver disease [2]. About $90 \%$ of perinatal infections become chronic and mortality in childhood is high. Nigeria belongs to the region of the world with hyperendemic prevalence $>8 \%$ [1]. In the South -southern part of Nigeria where this study was conducted, the prevalence among low risk pregnant women was $2.2 \%$ over a decade ago [3]. Currently, the prevalence, $4.3 \%$ double. Fortunately, HBV is vaccine-preventable and vaccination remains the most important strategy for its control. In 1981, Maurice Hilleman discovered the vaccine and vaccination was first introduced in 1982 [4]. The recombinant vaccine by Pablo DT Valenzuela in 1986 [5] replaced the earlier vaccine.

Vaccination has evolved into a comprehensive strategy for virtual elimination of vertical and horizontal transmission [6]. Vaccination of children against HBV was introduced by the Government of Nigeria (GON) into the National Program on Immunization (NPI) in 1995 [1] but became available in $2004[1,7,8]$. The pentavalent (five-inone) vaccine against diphtheria, tetanus, whooping cough, hepatitis $\mathrm{b}$ and hemophilus influenza type $\mathrm{b}$, all through a single dose [9], was officially introduced into the NPI 22 June 2012 [10]. The long delay in commencing the intervention and lack of national HBV surveillance program contributed to the hyperendemicity with seroprevalence of hepatitis B surface antigen (HBsAg) ranging between 10-40\% [1]. Mother-to-child transmission (MTCT) of HBV and its seromarkers can occur in utero, during intrapartum and postnatally to the infant $[6,11,12]$.

Intervention involving administration of hepatitis $B$ immunoglobulin (HBIG) and vaccine started at birth for exposed infants reduces the risk of MTCT to $<10 \%$ for $\mathrm{HBsAg} / \mathrm{HBeAg}$ positive mothers [11,12]. HBIG has high levels of antibody to HBsAg, immediately effective, and is protective for several months $[6,13]$. A

*Corresponding author: Onakewhor JUE, Department of Obstetrics and Gynecology, University of Benin Teaching Hospital, Benin City, Nigeria and Institute of Human Virology, University of Maryland School of Medicine, Baltimore, Maryland, United States of America, E-mail: jonakewhor@yahoo.com

Received July 30, 2013; Accepted September 18, 2013; Published September 22, 2013

Citation: Onakewhor JUE, Charurat M, Matthew O, Osagie E, Asemota MO, et al. (2013) Immunologic Pattern of Hepatitis B Infection among Exposed and Non-Exposed Babies in A PMTCT Program in Low Resource Setting: Does Every Exposed Newborn Require $200 \mathrm{IU}$ of Hepatitis B Immunoglobulin? J Vaccines Vaccin 4: 207. doi: 10.4172/2157-7560.1000207

Copyright: (c) 2013 Onakewhor JUE, et al. This is an open-access article distributed under the terms of the Creative Commons Attribution License, which permits unrestricted use, distribution, and reproduction in any medium, provided the original author and source are credited. 
Citation: Onakewhor JUE, Charurat M, Matthew O, Osagie E, Asemota MO, et al. (2013) Immunologic Pattern of Hepatitis B Infection among Exposed and Non-Exposed Babies in A PMTCT Program in Low Resource Setting: Does Every Exposed Newborn Require $200 \mathrm{IU}$ of Hepatitis B Immunoglobulin? J Vaccines Vaccin 4: 207. doi: 10.4172/2157-7560.1000207

Page 2 of 3

standard three-dose-course HBV vaccination is expected to produce adequate immune response (anti-Hbs antibody level $>100 \mathrm{mIU} / \mathrm{ml}$ ) one to four weeks later and provides protection for at least 25 years [14]. Full response to vaccination occurs in about $85-90 \%$ of individuals [15]. Others respond poorly with antibodies levels $<100 \mathrm{mIU} / \mathrm{ml}$ (i.e. 10 $\mathrm{iu}--100 \mathrm{mIU} / \mathrm{ml}$ ) and for whom booster dose is recommended $[6,15]$. Non-responders mount anti-Hbs antibody $<10 \mathrm{mIU} / \mathrm{ml}$ and current or past $\mathrm{HBV}$ infections may be risk factor. Preterm neonates with birth weight $<2000 \mathrm{~g}$ vaccinated at age $<1$ month [7] alcohol, smoking, obesity, age of 40 years, advanced liver disease, renal dialysis [16,17], and immuno-suppression including HIV [18] are risk factors for poor immune response. Newborns, health-care workers and diabetic patients are recommended for routine vaccinations [6]. When maternal antenatal anti-retroviral treatment is combined with appropriate infant vaccination, the rate of MTCT and vaccine non-response is reduced $[19,20]$. In spite of the improvements in the management of pregnant women, only about $50 \%$ of expectant mothers with HBsAg-positivity are identified [6].

In Nigeria, immunization coverage increases from about $15 \%$ in 1979 to $80 \%$ in 2007 [1].The GON with support from UNICEF routinely provides $\mathrm{HBV}$ vaccines at no cost to infants while couples provide HBIG for their exposed babies. In the absence of post-exposure immunoprophylaxis HBV-exposed newborns whose mothers are $\mathrm{HBsAg}^{+} / \mathrm{HBeAg}^{+}$have $70 \%--90 \%$ risk of having chronic infection by age 6 months [21] while $\mathrm{HBsAg}+/ \mathrm{HBeAg}$ - exposed infants have $<10 \%$ risk of having chronic infection in the absence of post-exposure immunoprophylaxis [22].

Antenatal screening for $\mathrm{HBsAg}$, treatment of women with $\mathrm{HBe}$ antigenemia and passive immunoprophylaxis with HBIG and active vaccination with $\mathrm{HBV}$ vaccine of exposed infants started June 15, 2005 in the Unit. The most daunting challenge has remained the prohibitive cost of HBIG. The costs of the standard $200 \mathrm{IU}$ for an average weight baby is seventy five thousand Nigerian Naira (NGN75, 000.00 or USD500.00; at official exchange rate of NGN150.00 = USD1.00). Procurement of multi-dose vials by the hospital Pharmacy Department to reduce cost has minimal effect with cost ranging between USD (466.67 -- 480.00) for 200 IU. With depreciating Naira value currently around NGN162.00 to USD1.00 is by implication USD540.00 for 200 IU HBIG. The minimum wage paid by the Federal (GON) is NGN18.000 (USD120.00) per month. Many state governments are unable/unwilling to pay this rate. Consequently, the standard intervention with 200iuHBIG for immunoprophylaxis for exposed newborns is prohibitive to many couples.

Routine prenatal screening for HBV infection and its management became part of routine care in this Unit June 15, 2005. The effectiveness of this intervention and the NPI at preventing infant HBV infection were yet to be evaluated. Data on MTCT of HBV in PMTCT programs in low resource settings is scarce, hence this pilot study.

\section{Objective}

The primary objective is to determine the immunological pattern of HBV seromarkers among HBV-infected women and exposed baby pairs in a cohort of pregnant women and the incidence of MTCT and seromarkers of HBV in out prevention program (a low resource setting).

Secondly, to compare immunological response of HBV-unexposed babies (Group A) who received standard dose of 200iu HBIG plus a course of 3 doses of HBV vaccine with exposed babies (Group B) who for financial constraint received 100iu HBIG plus a course of 3 doses of $\mathrm{HBV}$ vaccine and unexposed babies (control or Group C) who received a course of 3 doses of $\mathrm{HBV}$ vaccine.

\section{Hypothesis}

Where HBV infected mother is HBeAg--/HBcAbIgM-- there would be no difference in the rates of MTCT at 12 months of age between exposed babies who received standard dose 200iu of HBIG plus 3 doses of HBV vaccine and those who received 100iu HBIG plus 3 doses of $\mathrm{HBV}$ vaccine.

\section{Methodology}

\section{Study design, setting and subjects}

This was a prospective pilot observational study involved a cohort of consented HBV-infected pregnant women and their exposed babies and HBV-uninfected women-baby pairs as control. The study was carried out at the Department of Obstetrics and Gynecology, University of Benin Teaching Hospital, Benin City, Nigeria.

\section{Prenatal HBSAG testing protocol}

All pregnant women had routine are routinely screened for HBV infection (HBsAg) during the first antenatal visit pre-test counseling by trained counsellors in our prevention of mother-to-child transmission (PMTCT) of HIV/HBV Program. Hepatitis B infected women who gave consent were enrolled into the study, managed and mother-baby pairs followed up from 0-18 months. The controls (see above) were enrolled consecutively at the immunization clinic at ages 6 months and followed up for 18 months

Specifically, using aseptic technique, ten millilitre of blood was taken from the ante-cubital vein and sent in potassium ethylene diamine tetra acetic acid (EDTA)-anticoagulant tubes to the Haematology and Blood Transfusion Laboratory for screening. Sera obtained (after centrifugation at 3500rpm for 15 minutes) were analyzed for $\mathrm{HBsAg}$ using a rapid, direct, third generation immunochromatographic test for qualitative monoclonal and polyclonal antibodies to HBV (anti-HBsAg) (Clinotech ${ }^{\circledR}$ Diagnostics HBsAg detection; Clinotech Diagnostics \& Pharmaceuticals, Inc. Canada) [23]. The test uses the principle of direct binding double sandwich antibody (Ab-Ag-Ab) mechanism. It has no cross-reactivity with $\mathrm{HbeAg}, \mathrm{HbcAg}$ and $\mathrm{HbcAbIgM}, \operatorname{IgG}$ and $\operatorname{IgA}$ ) but capable of detecting all subtypes of HbsAg (adr,adw,ayr and ayw) [23]. Repeatedly reactive samples were subjected to further test using LumiQuick HBV-5 panel test based on immunochromatographic assay for rapid visual detection of markers of HBV including HBs-antigen, anti-HBs-antibodies, HBe-antigen, anti-HBe antibodies and anti$\mathrm{HBc}$ (IgG and $\operatorname{IgM}$ ) antibodies in accordance with the manufacturer (LumiQuick Diagnostics, Inc., Scott Blvd, Santa Clara, CA, USA)'s instructions [24]. All HBsAg reactive samples were confirmed using enzyme-linked immunosorbent assay (ELISA). In addition, screening for HCV and tuberculosis is undertaking to identify co-infections.

Viral load assays were done at the Human Virology Laboratory of the Nigerian Institute for Medical Research (NIMIR), Lagos Nigeria (one of the two national reference laboratories) using Cobas Taqman Roche Diagnostics, Switzerland with kind assistance from Roche Pharmaceuticals, Nigeria for air transport of specimens under cold chain.

\section{Unit protocol for managing $\mathrm{HBV}$ infected Women}

All HBsAg seropositive women are screened for HIV, Syphilis and HCV co-infections and evaluated for complete blood counts, liver and 
Citation: Onakewhor JUE, Charurat M, Matthew O, Osagie E, Asemota MO, et al. (2013) Immunologic Pattern of Hepatitis B Infection among Exposed and Non-Exposed Babies in A PMTCT Program in Low Resource Setting: Does Every Exposed Newborn Require $200 \mathrm{IU}$ of Hepatitis B Immunoglobulin? J Vaccines Vaccin 4: 207. doi: 10.4172/2157-7560.1000207

kidney functions, ultrasound scan and where feasible HBV viral load (VL). Liver biopsy, when considered to grade and stage liver disease for patients who meet the criteria for chronic hepatitis, is delayed till after delivery. Infected mothers and exposed babies are co-managed with gastroenterologists and paediatricians respectively.

Women that tested positive for HbsAg, HBe, HBcAbIgM and deranged liver enzymes (levels above the upper limit of normal for our laboratory reference values) are considered to have active viral infection irrespective of the viral load. They and especially those with high VL (HBV DNA levels $\geq 2,000 \mathrm{IU} / \mathrm{ml}$ ) are considered for antiretroviral prophylaxis with lamivudine $150 \mathrm{mg}$ b.d and tenofovir $300 \mathrm{mg}$ once daily from the third trimester till after delivery to reduce VL and prevent MTCT. Women seropositive for HBsAg but HBeAg--/ HBcAbIgM-- and have HBeAb, HBcAbIgG and VL $<2000 \mathrm{IU} / \mathrm{mL}$ are considered carriers with chronic infection and low risk for MTCT by Unit protocol. They are not placed on antiretroviral prophylaxis. Postpartum, all women are encouraged to breastfeed and mother-baby pairs followed-up.

Partner testing, treatment and vaccination, though part of routine care when indicated, is not part of this study. Uninfected women and exposed but HBsA-- partners had three doses of HBV vaccine; Engerix B ${ }^{\star}$ (GlaxoSmithKline Biologicals s.a, Rixensart, Belgium) using the immunization schedule of the NPI.

\section{Management of and HBV exposed babies}

Exposed babies had $3 \mathrm{ml}$ of cord blood collected at birth. Depending on age $2-5 \mathrm{ml}$ of peripheral blood were serially collected at age 6 weeks, 6, 9, 12 and 15 months into EDTA tubes for immunological testing to evaluate immune responses to hepatitis $\mathrm{B}$. The final blood collection was at age 12 months after completing third dose vaccination. Babies that did not respond to immunization (anti-HBsAg- but and $\mathrm{HBsAg}^{--}$) at 12 months were revaccinated and retested for HBs-antibodies at 1518 months of age using LumiQuick HBV-5 panel test as stated above. Where immediate analysis of specimen was not feasible, the sera were stored at $-81^{\circ} \mathrm{C}$. The babies were followed-up at the postnatal and immunization clinics.

\section{The unit immunoprophylaxis and immunization protocol}

All exposed babies receive HBIG (Bio Product Laboratories, Dagger Lane, Herts, UK) $200 \mathrm{IU}$ (at $40 \mathrm{IU} / \mathrm{kg}$ bodyweight) into anterolateral thigh muscle within 12 hours of birth. In this study, HBV exposed newborn babies were self-selected into receiving either the standard 200 IU or 100 IU HBIG based on parents choice and affordability. In addition, all medically stable infants are given three doses of HBV vaccine; Engerix $\mathrm{B}^{ø}$ (GlaxoSmithKline Biologicals s.a, Rixensart, Belgium). The first dose is given within 12 hours of birth into contralateral anterolateral thigh muscle. The second and third doses are given at 4 weeks and 6 months in accordance with the NPI protocol.

Exposed babies were followed-up at the Postnatal, Immunization and Child Welfare Clinics at 4weeks, 6, 9, 12, 15 and, where necessary, 18 months respectively. $\mathrm{HBsAg}^{-}$infants that did not mount antibodies at 9 and 12 months were revaccinated with a second 3-dose series and retested 1-2 months after the final dose of vaccine as practiced elsewhere [6]. As recommended by the CDC [25] and per the Unit protocol, exposed infants were breastfed beginning immediately after birth. Infected $\left(\mathrm{HBsAg}^{+}\right)$infants are appropriately managed by the paediatrician.

This routine care has been ongoing since June 2005 but immunological screening of mother-baby pairs using 5-panel tests commenced with this study in 2011. Maternal biodata were obtained using a questionnaire and labor and delivery data were extracted at delivery from the delivery records. For the controls, obstetric data were retrospectively obtained from the client's antenatal and delivery records including the Labor Ward Delivery and Postnatal Registers using their clients' hospital identifiers. The women and their babies were rescreened for HBsAg as detailed above.

\section{Outcome measures}

Incidence of MTCT of $\mathrm{HBV}$ (HBsAg, ${ }^{+} \mathrm{HbeAg}$ ), pattern of immunological response (anti-HBsAg, HBeAb, HBcAg (IgG/IgM) antibodies, and proportion of babies who responded to vaccination after passive/active immunoprophylaxis with HBIG and complete $\mathrm{HBV}$ vaccination were the main outcome measures.

\section{Ethical issues}

Ethical approval was obtained from the University of Benin Teaching Hospital Ethics' Committee. All adult subjects gave informed consent prior to their enrolment while either or both parents gave consent for enrolment of their babies. We declare no conflict of interest. The study team funded the cost of screening for HBV.

\section{Data Analysis}

The data were analyzed using GraphPad Instat3, version 3.06, 32 bit for windows, 2003 software (GraphPad Software Inc. Camino Real, San Diego, USA). The immunological response to vaccination between groups was compared using Chi-squared $\left(\chi^{2}\right)$ and Fisher's tests were appropriate. $P$ value $\leq 0.05$ was considered significant. Student's $t$ test was used for independent variables and $\mathrm{P}$ value $\leq 0.05$ was considered significant at 95\% Confidence Interval.

\section{Results}

There were $65 \mathrm{HBV}$ infected $\left(\mathrm{HBsAg}^{+}\right)$women but $45(69.2 \%)$ and all $65 \mathrm{HBsAg}$ controls have complete information for analysis. Three women (one $\mathrm{HBsAg}^{+}$and $2 \mathrm{HBsAg}$ control groups) reported previous blood transfusion. None of the women had previous vaccination against HBV and used intravenous drugs. The time of HBV diagnosis among infected women is shown in table 1 while the maternal and neonatal HBV immunological patterns at birth and during follow-up are shown in tables 2 and 3 respectively.

Partners testing showed serodiscordance of 1:6. Of 13/45 (28.9\%) partners that responded to invitation and tested of whom 2/13 (15.4\%) were $\mathrm{HBsAg}^{+}$. Majority, $84.7 \%$ were seronegative. The spouses of the 2 seropositive men cleared their HBsAg and were seronegative at six months and one year.

All except $2(3.0 \% ; 2 / 65)$ women tested negative for HIV coinfection. One (1.5\%) had HBV/HIV co-infection. The baby was HBsAg and responded to vaccination. The other woman had triple HBV/ HIV/ TB co-infections. She relocated to another West African (her) country. She and 4 other $\mathrm{HBV}^{+}$mothers and exposed babies were for various

\begin{tabular}{|c|c|c|}
\hline Time of HBV Diagnosis & frequency & Per cent \\
\hline Past (before index pregnancy) & 6 & 13.3 \\
\hline First Trimester & 2 & 4.4 \\
\hline Second trimester & 30 & 66.7 \\
\hline Third trimester & 7 & 15.6 \\
\hline Total & 45 & 100 \\
\hline
\end{tabular}


Citation: Onakewhor JUE, Charurat M, Matthew O, Osagie E, Asemota MO, et al. (2013) Immunologic Pattern of Hepatitis B Infection among Exposed and Non-Exposed Babies in A PMTCT Program in Low Resource Setting: Does Every Exposed Newborn Require $200 \mathrm{IU}$ of Hepatitis B Immunoglobulin? J Vaccines Vaccin 4: 207. doi: 10.4172/2157-7560.1000207

Page 4 of 3

reasons lost to follow-up and excluded from the study making for attrition rate of $7.7 \%$. The other 15 exposed babies, for various logistics reasons, were yet to have their immune status determined. They are not included in the analysis. Thus, data for 45(69.2\%) $\mathrm{HBsAg}^{+}$mothers and 46 exposed babies and the control group of all $65 \mathrm{HBsAg}^{-}$mothers and 66 babies were available for analysis (Tables 2 and 3).

The median age booking was 21 weeks and mean gestational age at antenatal diagnosis was $20.5 \pm 7.8$ weeks (range 6-35 weeks); 95CI $17.12-23.90$ (Table 1). The mean for past diagnosis was $5.6 \pm 3.0$ (range 2-9) years.

The mean age of HBV infected women was $30 \pm 15$ (range 2341) years $(95 \% \mathrm{CI} 28.63$ - 31.68) vs. $38.613 \pm 2.25$ (range $26-41$ ) years 95\%CI 38.05-39.17; $\mathrm{P}<0.0001$. HBV infected women were younger. There was no statistically significant difference between positives and negatives for mean and median parity $\mathrm{p}=0.99$; mean gravidity $\mathrm{p}=0.39$, and mean gestational age at delivery $(\mathrm{GAD}) \mathrm{p}=0.80$. The median $\mathrm{GAD}$ was 40 weeks for both groups.

$\mathrm{HBV}^{+}$women were delivered more by caesarean sections $(\mathrm{C} / \mathrm{S})$; $4 / 45(4.4 \%)$ vs. $1 / 65(6.1 \%)$ for negatives; $\mathrm{p}=0.0001$. Indications in two were for obstetric reasons and one woman had HBV/HIV co-infection. The baby was negative for both infections. There was no difference in mean birth weights $3.35 \pm 0.53 \mathrm{~kg}$ (Range $2.5-4.3 \mathrm{~kg}$ ); $95 \%$ CI $3.19-3.51$ for positives vs. $3.60 \pm 0.72 \mathrm{~kg}$; $95 \%$ CI 3.38-3.81 and negatives; $\mathrm{P}=0.06$. The median weights were $3.20 \mathrm{~kg}$ and no low birth weights. The mean first and 5 minutes Apgar's scores were $8.0 \pm 0.4 \mathrm{p}=0.35$ and $9.0 \pm 0.23$ vs. $8.9 \pm 0.17 \mathrm{p}=0.03$ for exposed and non-exposed babies respectively.

Two women cleared their antigens and were seronegative at 6 and 12 months (table 2) were considered potentially infective for MTCT and were so managed to enhance PMTCT. All babies were HBsAg and HBeAg-seronegative. The incidence of MTCT was zero $(0.0 \%)$ irrespective of the dose of HBIG administered. All babies had complete vaccination and were breastfed.

Due to high cost of viral load (VL) test (NGN28,000 or USD186.7 per assay), $7(15.6 \%)$ women had VL assay with a mean of 1299.96 $\pm 1045.2 \mathrm{IU} / \mathrm{ml}$ (Range 43.0 - $2403.0 \mathrm{IU} / \mathrm{ml}$ ); 95\%CI 202.16-2396.2 and a median value of 1310.5 . $K S=0.25$. All except two women had normal liver enzymes.

There was no significant difference in the immunological pattern of the babies at 6 and 9 months (table not shown). Seven and 10 babies were being expected at 9 and 12 months appointments respectively for rescreening. The overall rate of non-response to vaccination was $8.0 \%$. The incidence of 'non-responders' at 12 months was $17.1 \%$ vs. $3.0 \%$; $\mathrm{p}<0.01$ (RR 0.42; 95\% CI 0.25-0.069) for exposed infants and nonexposed infants respectively.

Of the 8 babies ( 6 for exposed and 2 non-exposed; table 3) who did not mount qualitatively detectable anti-HBsAg antibodies (nonresponders), six have been revaccinated while 2 were being expected. Of those revaccinated $4(66.7 \%)$ have mounted antibodies while 2 were yet to complete the revaccination process. Among exposed babies, about a third (28.6\%) had HBcAb antibodies at one year. Mothers of nonexposed babies that failed to mount antibodies and all non-responders were retested for HBsAg and were all negative.

\section{Discussion}

This pilot study has highlighted the need to evaluate our immunization programs especially for $\mathrm{HBV}$-exposed infants. Though the sample size was small (a pilot), the rate of MTCT for HBsAg and HBeAg were $0.0 \%$ irrespective of the dose of HBIG administered for prophylaxis. An MTCT rate of 3\% was reported for Sydney women [26] and $65 \%$ for Indian women with $\mathrm{HBeAg}^{+}$and $\mathrm{HBV}$ DNA [27]. $\mathrm{HBeAg}$ is a risk factor for MTCT but only $3.0 \%$ of the women in our study were seropositive. The prevalence is also lower than the $12.5 \%, 29 \%$ and 56.8\% reported for Turkish,[12] Sydney [26] and Indian women respectively while the anti-HBe of $77.8 \%$ in the study was comparable to the $77.5 \%$ reported for same Turkish population [12].

Hepatitis B infected women were generally younger than noninfected controls; $\mathrm{p}<0.0001$. Similar finding was reported among Indian women [27]. We could find no reliable explanation for this observation. However, younger women are more vulnerable than older ones to

\begin{tabular}{|c|c|c|c|}
\hline \multirow{2}{*}{ Indicator (Antigen/antibodies) } & \multicolumn{2}{|c|}{ HBV Positive women(N= 45 ) } & \multicolumn{2}{|c|}{ HBV Exposed infants At birth n=46** } \\
\cline { 2 - 4 } & Positive (\%) & Negative (\%) & 0 \\
\hline HbsAg & 43 & $2(4.4)^{\star}$ & 46 \\
\hline HBsAb & $2(4.4)$ & 43 & $4(8.7)$ \\
\hline HBeAg & $1(2.2)$ & 44 & 0 \\
\hline HBeAb & $35(77.8)$ & 10 & $12(26.1)$ \\
\hline HBcAb IgG & $42(93.3)$ & 3 & $40(86.9)$ \\
\hline
\end{tabular}

*2women with $\mathrm{HBsAg}+$ cleared their antigens and were seronegative at 6 and 12 months. Their Partners were HBsAg. ${ }^{* *}$ One set of twins.

Table 2: Patterns of Maternal and Neonatal HBV Immunology at Birth.

\begin{tabular}{|c|c|c|c|c|c|c|c|c|c|}
\hline \multirow{3}{*}{$\begin{array}{l}\text { Indicator (Antigen/ } \\
\text { antibodies) }\end{array}$} & \multicolumn{6}{|c|}{ HBV Exposed infants at } & \multirow{2}{*}{\multicolumn{2}{|c|}{$\begin{array}{c}\text { Un-Exposed infants } \\
\text { Age } 12 \text { months } n=66(\%)\end{array}$}} & \multirow{3}{*}{$\begin{array}{c}\text { Non-responders at } 12 \\
\text { months }(\%)\end{array}$} \\
\hline & \multicolumn{2}{|c|}{ Birth $n=46(\%)$} & \multicolumn{2}{|c|}{ Age 9 months $n=39$} & \multicolumn{2}{|c|}{ Age 12 months $n=35(\%)$} & & & \\
\hline & pos & neg & pos & neg & pos & neg & pos & Neg & \\
\hline $\mathrm{HbsAg}$ & 0 & 46 & 0 & 39 & 0 & 35 & 0 & 66 & NA \\
\hline $\mathrm{HBsAb}$ & $4(8.7)$ & $42(91.3)$ & $34(87.2 \%)$ & 5 & $29(82.9 \%)$ & $6(17.1)$ & $64(97.0)$ & $2(3.0)$ & $8(8.0)$ \\
\hline $\mathrm{HBeAg}$ & 0 & 45 & 0 & 39 & 0 & 35 & 0 & 66 & NA \\
\hline $\mathrm{HBeAb}$ & $12(26.1)$ & $34(73.9)$ & $6(15.4)$ & 39 & 0 & 35 & 0 & 66 & NA \\
\hline $\mathrm{HBcAb} \lg \mathrm{G}$ & $40(86.9)$ & $6(13.0)$ & $20(51.2)$ & 19 & $1(2.8)$ & 34 & 0 & 66 & NA \\
\hline $\mathrm{HBcAb} \lg \mathrm{M}$ & 0 & 46 & 0 & 39 & 0 & 35 & 0 & 66 & NA \\
\hline
\end{tabular}

$\mathrm{NA}=$ Not applicable

Table 3: Exposed and Non-Exposed Infants' Immunologic Pattern for HBV Seromarkers. 
Citation: Onakewhor JUE, Charurat M, Matthew O, Osagie E, Asemota MO, et al. (2013) Immunologic Pattern of Hepatitis B Infection among Exposed and Non-Exposed Babies in A PMTCT Program in Low Resource Setting: Does Every Exposed Newborn Require $200 \mathrm{IU}$ of Hepatitis B Immunoglobulin? J Vaccines Vaccin 4: 207. doi: 10.4172/2157-7560.1000207

Page 5 of 3

acquire HIV infection and social behaviour have been implicated $[3,28]$. Both infections have common mode of transmission. We had earlier reported the prevalence of HBV infection among our pregnant population to be $2.2 \%$ [3] and a decade later $4.3 \%$ (Onakewhor et al. [3] West African Journal of Medicine - in press). Though anti-HBc appears at the onset of symptoms or liver enzyme derangement in acute HBV infection and persists for life, [6] 3(6.7\%) women lost the antibodies (table 3). We could find no reasons for this observation but one of the women belong to the two that cleared their HBsAg. There was high serodiscordance. Only 15.4\% (1:6) spouses of HBV-infected women were seropositive. Two women whose partners were HBsAg positive cleared their antigens. This makes partner testing and vaccination of uninfected spouses imperative.

The better 5 minutes Apgar's score $(\mathrm{p}=0.03)$ observed among exposed newborns may be misleading. This was probably a chance finding attributable to the mode of delivery rather than to HBV exposure status. $\mathrm{HBV}$ is not known to improve fetal hypoxia. More exposed babies were delivered by $\mathrm{C} / \mathrm{S}$ than controls; $\mathrm{p}=0.0001$ which is beneficial in preventing MTCT [27]. Similar observation was noted in an Indian study [27]. All babies were breastfed and none was infected confirming the recommendation that breastfeeding was not contraindicated among exposed infants $[1,6]$. Though, low birth weight infants have been reported to be commoner among mothers with acute HBV infection during pregnancy [6], all women in this study had normal birth weight babies including the woman with immunological evidence of acute infection ( $\mathrm{HBeAg}+$ ).

The overall incidence of non-response to vaccination of $8.0 \%$ in this study is comparable to the $6.7 \%$ non-responders reported among Brazilian women with HIV/HBV co-infection [29]. HIV/ $\mathrm{HBV}$ co-infection 29 and preterm infants weighing $<2,000 \mathrm{~g}$ at birth administered with HBV vaccine before age one month [6], are risk factors for poor infant response to vaccination. There were no low birth weight infants and the HIV/HBV-exposed infant mounted antibodies. However, HBV-exposed were less likely than the non-exposed babies to mount qualitatively detectable anti-HBs antibodies at one year (Table 3); $<<0.03$. We are aware that standard testing to determine immune response requires quantitative assay for anti-HBs levels and levels $>10 \mathrm{mIU} / \mathrm{ml}$ is considered adequate immunity $[6,15]$. However, we lacked facilities at the moment to do this test hence the qualitative method employed.

The pattern of maternal to fetal transfer and acquisition of immunoglobulins is shown in table 3. Except for HBsAg, $\mathrm{HBeAg}$ and $\mathrm{HBcAb}-\mathrm{IgM}$ transmission that was zero $(0.0 \%)$, it revealed no homogenous pattern of immunoglobulins transfer. For example at birth, the proportions of babies that had $\mathrm{HBsAb}, \mathrm{HBeAb}$ and $\mathrm{HBcAb}-$ IgG were $8.7 \%, 26.1 \%$ and $86.9 \%$ compared with $4.4 \%, 77.8 \%$ and 93.3\% respectively for the mothers. Only $23.0 \%$ and $15.4 \%$ mothers transmitted their $\mathrm{HBeAb}$ to their babies at birth and at 9 months respectively which were cleared by age 12 months (Tables 2 and 3). The baby that had HBcAb-IgG was retested for HBsAg and was seronegative but mother-baby pair was more difficult to follow-up [30].

Though immunoprophylaxis with 200 IU HBIG followed by standard vaccination can prevent MTCT by $95 \%$ [31], only $10.9 \%$ (5/46) parents afforded the cost of the standard 200 IU HBIG and the babies were so immunized plus complete vaccination. Due to financial constraints the majority, $82.6 \%(38 / 46)$ received 100 IU while $6.5 \%$ receive none. The overall MTCT of HBsAg was $0.0 \%$. In a similar study in China, only $53.3 \%$ exposed children received HBIG with MTCT rates of $2.4 \%, 3.1 \%$, and $71.6 \%$ for $\mathrm{HBsAg}^{+}, \mathrm{HBsAg}^{-} / \mathrm{anti}-\mathrm{HBc}^{+}$, and
anti-HBs ( $\geq 10 \mathrm{mIU} / \mathrm{ml}$ ) respectively for the children 1-7-year-old [32]. Neonates that received HBIG, anti-HBs is said to be detectable from the immunoglobulin if testing was done before age $<9$ months and passively acquired maternal anti-HBc from $\mathrm{HBV}$-infected mothers may persist and be detectable in infants up to age 24 months [6]. Consequently, serial following up testing for these immunoglobulins is ongoing. Where parents could not afford HBIG for their infants honouring appoints become more difficult.

High maternal VL $>200,000 \mathrm{iu} / \mathrm{mL}$ was a risk factor reportedly associated with $8 \%--30 \%$ MTCT rate in an American study [32] Two women with very high VL are on antiretroviral drugs, were yet to deliver and not included in the analysis. It is reported that exposed children who are not infected at birth remain at risk from long-term interpersonal contact with their infected mothers. Consequently, exposed babies were being followed-up until adequate immune response was obtained $[6,25]$.

\section{Limitations of the Study}

The small sample size (a pilot study), inability to provide every exposed new born with HBIG for prophylaxis due to prohibitive cost and lack of facilities on site for VL and quantitative assays for antiHBs were some of the limitations of the study. Also, due to logistic difficulties of long term follow-up and to mitigate possible high attrition rate, the control group was chosen from among women who delivered at periods at variance with that of the study subjects. Nevertheless, the objectives of the study were not derailed by these limitations.

\section{Conclusion}

The MTCT rate for HBV appears low ( $0.0 \%$ in this study) There was no significant difference in response to $\mathrm{HBV}$ immuno-protection between babies that receive 200iu HBIG dose compared to the group 100iu HBIG among exposed babies whose mothers show no evidence of active viral replication. The overall rate of non-response to vaccination was high with exposed infants less likely than unexposed to mount antibodies after immunoglobulin prophylaxis and complete vaccination. There is no homogenous pattern of maternal to fetal transfer of HBV seromarkers. We advocate provision of HBIG for exposed babies as integral part of care in the NPI program. Capacity for VL and quantitative anti-HBsAb assay be made available on site and mitigate the prohibitive cost of these tests and engender quality care. Multicenter studies are required to evaluate the new intervention before policy change.

\section{Acknowledgement}

We are grateful to the women and their spouses who not only participated but gave permission for the enrolment of their babies into this study. We sincerely appreciate the invaluable assistance of the Roche Pharmaceutical, Nigeria for providing cold chain and airlifting study specimen from the Study Center to NIMIR, Lagos for analysis and return of results. Furthermore, we are grateful to NIMIR for the laboratory assistance. Finally, we appreciate the staff of the Laboratory Department of the University of Benin Teaching Hospital for laboratory analysis of specimens and preparing others for shipment

\section{References}

1. Forbi JC, Vaughan G, Purdy MA, Campo DS, Xia GL, et al. (2010) Epidemic history and evolutionary dynamics of hepatitis $B$ virus infection in two remote communities in rural Nigeria. PLoS One 5: e11615.

2. Zanetti AR, Van Damme P, Shouval D (2008) The global impact of vaccination against hepatitis B: a historical overview. Vaccine 26: 6266-6273.

3. Onakewhor JU, Offor E, Okonofua FE (2001) Maternal and neonata seroprevalence of hepatitis B surface antigen (HBsAg) in Benin City, Nigeria. $J$ Obstet Gynaecol 21: 583-586. 
Citation: Onakewhor JUE, Charurat M, Matthew O, Osagie E, Asemota MO, et al. (2013) Immunologic Pattern of Hepatitis B Infection among Exposed and Non-Exposed Babies in A PMTCT Program in Low Resource Setting: Does Every Exposed Newborn Require $200 \mathrm{IU}$ of Hepatitis B Immunoglobulin? J Vaccines Vaccin 4: 207. doi: 10.4172/2157-7560.1000207

4. CDC (2009) Atlanta, Georgia: Centers for Disease Control and Prevention.

5. Anna C (2012) World Hepatitis Day: The History of the Hepatitis B Vaccine. Planned Parenthood Advocates of Arizona.

6. CDC (2006) A Comprehensive Immunization Strategy to Eliminate Transmission of Hepatitis B Virus Infection in the United States. MMWR 55: 16.

7. Sadoh AE, Eregie CO (2008) Age at presentation for infant immunization in Nigeria: implications for hepatitis B immunization. Public Health 122: 13181320.

8. World Health Organization (2005) WHO vaccine preventable diseases monitoring system 2005 global summary. Immunization profile-Nigeria.

9. Bar-On ES, Goldberg E, Hellmann S, Leibovici L (2012) Combined DTP-HBVHIB vaccine versus separately administered DTP-HBV and HIB vaccines for primary prevention of diphtheria, tetanus, pertussis, hepatitis $B$ and Haemophilus influenzae B (HIB). Cochrane Database Syst Rev 4: CD005530.

10. World Health Organization (2012) Nigeria Launches Penta Vaccine.

11. André FE, Zuckerman AJ (1994) Review: protective efficacy of hepatitis B vaccines in neonates. J Med Virol 44: 144-151.

12. Uyar Y, Cabar C, Balci A (2009) Seroprevalence of Hepatitis B Virus among Pregnant Women in Northern Turkey. Hep mon 9: 146- 149.

13. Anderson TA, Wexler DL (2005) States report hundreds of medical errors in perinatal hepatitis B prevention. St. Paul, MN: Immunization Action Coalition.

14. Van Damme P, Van Herck K (2007) A review of the long-term protection after hepatitis A and B vaccination. Travel Med Infect Dis 5: 79-84.

15. Joint Committee on Vaccination and Immunisation(2006) Immunisation Against Infectious Disease: The Green Book. Edinburgh: Stationery Office.

16. Rosman AS, Basu P, Galvin K, Lieber CS (1997) Efficacy of a high and accelerated dose of hepatitis $B$ vaccine in alcoholic patients: a randomized clinical trial. Am J Med 103: 217-222.

17. Pasricha N, Datta U, Chawla Y, Singh S, Arora SK, et al. (2006) Immune responses in patients with HIV infection after vaccination with recombinant Hepatitis B virus vaccine. BMC Infect Dis 6: 65

18. M Landrum, K Huppler Hullsiek, N Crum-Cianflone (2010) Hepatitis B Vaccine Response Predicts Progression to Clinical AIDS or Death. 17th Conference on Retroviruses \& Opportunistic Infections.

19. EASL (2009) Combined Maternal Treatment and Infant Vaccination Effective in Reducing Mother-to-Child HBV Transmission. Medscape.

20. Centers for Disease Control and Prevention (CDC) (2011) Use of hepatitis B vaccination for adults with diabetes mellitus: recommendations of the Advisory Committee on Immunization Practices (ACIP). MMWR Morb Mortal Wkly Rep 60: $1709-1711$.

21. Wong VC, Ip HM, Reesink HW, Lelie PN, Reerink-Brongers EE, et al. (1984) Prevention of the HBsAg carrier state in newborn infants of mothers who are chronic carriers of $\mathrm{HBsAg}$ and $\mathrm{HBeAg}$ by administration of hepatitis-B vaccine and hepatitis-B immunoglobulin: double-blind randomised placebo-controlled study. Lancet 1: 921-926.

22. Stevens CE, Toy PT, Tong MJ, Taylor PE, Vyas GN, et al. (1985) Perinatal hepatitis $B$ virus transmission in the United States. Prevention by passiveactive immunization. JAMA 253: 1740-1745.

23. Insert Clinotech Diagnostics \& Pharmaceuticals, Inc. 2101-11871 Horse Way Richmond BC V7A 5 H5 Canada.

24. Insert 2006-2012 LumiQuick Diagnostics, Inc. Scott Blvd, Santa Clara, CA USA)'s instructions.

25. CDC (2002) General recommendations on immunization: recommendations of the Advisory Committee on Immunization Practices (ACIP) and the American Academy of Family Physicians (AAFP). MMWR 51: 1-35

26. Wiseman E, Fraser MA, Holden S, Glass A, Kidson BL, et al. (2009) Perinata transmission of hepatitis B virus: an Australian experience. Med J Aust 190: 489-492.

27. Dwivedi M, Misra SP, Misra V, Pandey A, Pant S, et al. (2011) Seroprevalence of hepatitis $B$ infection during pregnancy and risk of perinatal transmission. Indian J Gastroenterol 30: 66-71.

28. Offor E, Onakewhor JU, Okonofua FE (2000) Maternal and neonatal seroprevalence of human immunodeficiency virus antibodies in Benin City, Nigeria. J Obstet Gynaecol 20: 589-591.

29. Abramczuk BM, Mazzola TN, Moreno YMF, Zorzeto TQ, Quintilio W, et al. (2011) Impaired Humoral Response to Vaccines among HIV-Exposed Uninfected Infants. Clinical and Vaccine Immunology 18: 1406-1409.

30. Linder N, Vishne TH, Levin E, Handsher R, Fink-Kremer I, et al. (2002) Hepatitis $B$ vaccination: long-term follow-up of the immune response of preterm infants and comparison of two vaccination protocols. Infection 30: 136-139.

31. Pan CQ, Duan ZP, Bhamidimarri KR, Zou HB, Liang XF, et al. (2012) An algorithm for risk assessment and intervention of mother to child transmission of hepatitis B virus. Clin Gastroenterol Hepatol 10: 452-459.

32. Chen X, Chen J, Wen J, Xu C, Zhang S, et al. (2013) Breastfeeding is not risk factor for mother-to-child transmission of hepatitis B virus. PLoS One 8: e55303. 